\title{
Tympanostomy tube otorrhea in children: prevention and treatment.
}

Van Dongen $T M A^{1}$, Damoiseaux RAMJ', Schilder $A G M^{1,2}$.

Author affiliations:

1 Julius Center for Health Sciences and Primary Care, University Medical Center, Utrecht, The Netherlands.

2 EvidENT, Ear Institute, University College London, London, United Kingdom.

Corresponding author:

Thijs MA van Dongen, MD, PhD; tel: +31611499973; email: tmavandongen@gmail.com.

Postal address: Julius Center for Health Sciences and Primary Care, STR 6.131, University Medical Center Utrecht, PO Box 85500, 3508 GA Utrecht, The Netherlands. 


\section{Abstract}

Purpose of review

One in two children treated with tympanostomy tubes experience episodes of otorrhea whilst their tubes are in place. In this review we present the results of the most recent publications on prevention and treatment of tympanostomy tube otorrhea (TTO).

\section{Recent findings}

Recent systematic reviews on water precautions for children with tympanostomy tubes support the American Academy of Otolaryngology - Head and Neck Surgery guideline recommendation against such preventive measures as there is no evidence that it protects against TTO.

Studies on tympanostomy tube design and material suggest that silicone tubes have a lower TTO risk and that biofilms appear to be mainly located in the perpendicular junction of the T-tubes and the round rims of the Paparella-type tubes. Another study shows that the biofilm-component DNAB-II protein is present in otorrhea of half of children with TTO. Targeting this protein could lead to a collapse of the biofilm structure and as such a potential new treatment for chronic TTO.

New systematic reviews show that antibiotic eardrops are the most effective first-line treatment of acute TTO and suggest that an antibiotic-corticosteroid combination is more effective than antibioticonly. Although in many countries quinolone eardrops are the preferred choice due to being nonototoxic, one study found a higher risk of persistent perforation after tube extrusion in children treated with quinolone eardrops as compared to children treated with aminoglycoside eardrops.

\section{Summary}

Recent evidence confirms that water precautions for children with tympanostomy tubes are not effective in preventing TTO. Antibiotic-corticosteroid eardrops are the most effective treatment of acute TTO. 
Key words

Otorrhea, tympanostomy tube, treatment, risk factors, review. 


\section{Introduction}

Tympanostomy-tube placement is one of the most frequently performed surgical procedures in children worldwide. ${ }^{1}$ It's main indications are recurrent acute otitis media (rAOM) and persistent otitis media with effusion (pOME). Tympanostomy tube otorrhea (or ear discharge) (TTO) is a common problem in children with tubes; one in two children experience one or more TTO episodes. ${ }^{2,3}$ TTO is usually caused by otitis media, when middle ear fluid drains through the tube. It can be accompanied by foul odor, pain, and fever and as such can affect the child's quality of life. ${ }^{4}$ Acute tympanostomy tube otorrhea (ATTO) is defined as otorrhea for up to 2 to 8 weeks; when otorrhea persists beyond that it is defined as chronic tympanostomy tube otorrhea (CTTO). Postoperative TTO, occurring in the first 2 weeks after tympanostomy tube placement, is thought to be associated with the surgery or the condition of the middle ear during surgery, rather than with a new infection.

It is known that younger children and those receiving tubes for rAOM are at higher risk of TTO, and so are those with a recent history of recurrent upper respiratory tract infections and having older siblings. ${ }^{2}$ Reports on the benefit of water precautions to prevent TTO have been inconsistent. ${ }^{5}$ In 2014 the first head to head comparison of the three most common approaches to the treatment of ATTO, antibiotic-corticosteroid eardrops, oral antibiotics and initial observation, showed that treatment with eardrops was by far most effective both clinically and economically. ${ }^{6,7}$ Current guidelines recommend topical antibiotics in children with ATTO. ${ }^{8,9}$

The objective of this review is to present the most recent evidence on prevention and management of TTO. We selected studies published between June 2016 and April 2018 and discuss their implications for clinical practice. 


\section{Prevention}

New studies on prevention of TTO focused on water exposure, tube design and tobacco exposure.

\section{Water exposure}

Many physicians still recommend water precautions for children with tympanostomy tubes despite recent guidelines discouraging this practice..$^{8,10,11}$ In January 2016, shortly before our search window, but relevant to this topic, Moualed et al. published a Cochrane Review including 2 RCTs of the effectiveness of water precautions for prevention of TTO. ${ }^{5}$ The first trial included 201 participants and showed that wearing ear plugs reduces TTO incidence by only 0.36 episodes per year $(95 \% \mathrm{Cl}$ 0.45 to -0.27$)$. The second trial included 212 children and found no difference in TTO incidence between those instructed not to swim or submerge their heads while bathing and those who did not receive these instructions. A 2017 systematic review, commissioned by the US Agency for Healthcare Research and Quality and also including non-randomized studies, showed similar results. ${ }^{12}$ Finally, a 2017 retrospective study comparing 116 children with tubes who were prescribed earplugs for water protection to 27 children who did not receive earplugs neither showed a benefit regarding TTO incidence or quality of life. ${ }^{13}$

Ricks et al. and conducted a laboratory experiment looking at water penetration of fresh, soapy, chlorinated and sea water through four different tube types: Shah, mini-Shah, T-tube and titanium bobbin. ${ }^{14}$ The mini-Shah tube appeared to provide the best protection against water penetration for all aqueous solutions. Using the same model with the Shah-tube and to inform patients who wish to dive, Ibrahim and colleagues compared the hydrostatic head required for water to pass through the tube. ${ }^{15}$ They showed that soapy water required the lowest pressure to penetrate the Shah-tube, distilled water the highest, and found no difference between chlorinated and sea water.

\section{Tube design}


In 2016, Tamir et al. demonstrated that in vitro growth of Pseudomonas aeruginosa biofilms on tympanostomy tubes was mainly located in the perpendicular junction between the body and the flanges of the T-tubes and in the round rims of the Paparella-type tubes. ${ }^{16}$ These zones can be future target areas for changes in design or antibiofilm coatings of tubes to prevent recurrent or chronic TTO.

In 2018, Knutsson et al. published follow-up data of their RCT of tympanostomy tubes of various materials and shapes: fluoroplastic-short, silicone-short, fluoroplastic-long, silicone-long. ${ }^{17}$ They randomized 378 children per ear to either one of these tubes so that most children received different tubes in both ears. Time to first TTO episode was significantly longer for silicone tubes than fluoroplastic tubes; the hazard ratio did not change when adjusted for tube shape.

\section{Tobacco exposure}

In a retrospective chart review of 191 children with tubes, Bizzell at al. studied the effects of tobacco exposure on occurrence of TTO. ${ }^{18} \mathrm{~A}$ multivariable regression analysis with correction for age, preoperative diagnosis, operative findings and duration of follow-up showed that exposure to tobacco smoke was independently associated with TTO occurrence (odds ratio $=2.307 ; 95 \%-\mathrm{Cl}: 1.734$ -6.028). Although we would recommend counseling parents regarding the risks of exposing their children to tobacco smoke in general, in the context of prevention of TTO the evidence is still up for debate; this new study did not include relevant confounders and another previous study did not find an independent association between tobacco exposure and TTO occurrence. ${ }^{2}$

\section{Treatment}

Recent publications include RCT's of novel topical drugs to prevent postoperative TTO or treat ATTO, systematic reviews on ATTO treatment, and studies on a potential new target for treatment of biofilms in CTTO and on the complications of quinolone eardrops. 


\section{Postoperative TTO}

The effectiveness of a single injection of a ciprofloxacin otic suspension into the middle ear during tube placement in preventing postoperative TTO has been studied in three recent RCTS.${ }^{19,20}$ Park et al. present the results of two double-blind phase 3 trials including a total of 532 patients aged 6 months to 17 years who received the injection perioperatively versus tympanostomy tubes alone. ${ }^{19}$ The cumulative proportion of TTO on day 15 was lower in the treatment than in the control group: $23.0 \%$ vs $45.1 \%$, age-adjusted odds ratio, $0.341 ; p<0.001)$. This latter figure is much higher than the incidence of postoperative TTO reported in observational studies, that is $10-20 \%$, which could be due to the close monitoring of participants in a trial setting. ${ }^{2,3}$ Occurrence of adverse events including pyrexia and postoperative pain was similar in both groups.

Dohar et al. present data on this new drug's safety and postoperative TTO incidence in a 'real-world pediatric' cohort of 501 children who received the single intratympanic injection of ciprofloxacin otic suspension during tube placement. ${ }^{20}$ The safety profile was similar as in the trials, but the postoperative TTO incidence was with $8.8 \%$ much lower than the proportion found in the beforementioned phase-3 trials.

ATTO

Recent systematic reviews of studies on the effectiveness of various treatments for ATTO have come to the conclusion that antibiotic eardrops are most effective; regarding antibiotic-corticosteroid eardrops conclusions vary.

In 2016, Venekamp et al. updated the Cochrane Review on management of ATTO. ${ }^{21}$ They included nine RCTs with a total of 2132 children children who developed TTO at least two weeks after tube placement; they found moderate to low-quality evidence that antibiotic eardrops (with or without a corticosteroid) are more effective than oral antibiotics, corticosteroid eardrops and no treatment in children with ATTO. 
Steele et al. performed a network meta-analysis providing estimates for all pairwise comparisons of management strategies, including those that have not been directly compared in the RCT's they included. ${ }^{12}$ Based on data from seven RCT's, they found a probability of $77 \%$ that in daily practice antibiotic-corticosteroid eardrops are superior over antibiotic only eardrops, oral antibiotics or no treatment. Probabilities are $22 \%$ for antibiotic-only eardrops, $1 \%$ for oral antibiotics and $0 \%$ for no treatment.

In 2017, Spektor et al. published two double-blind RCT's (unpublished data of one of these was included in the Cochrane Review) of a new antibiotic-corticosteroid eardrop; ciprofloxacin/fluocinolone. ${ }^{22} 662$ children with TTO for up to 3 weeks were randomized to the new eardrops or ciprofloxacin only or fluocinolone only eardrops. Time to cessation of otorrhea was 4.23 days in patients receiving ciprofloxacin/fluocinolone eardrops compared to 6.95 days in those receiving ciprofloxacin only and longer than 22 days (total duration of follow-up) in those receiving fluocinolone only drops. They conclude that the combination of an antibiotic-corticosteroid eardrop is more effective than an antibiotic only eardrop with regard to time to resolution of otorrhea.

Previous studies showed that antibiotic eardrops reach very high drug concentrations in the otorrhea and low or absent serum drug concentrations and are therefore less likely to cause antimicrobial resistance as compared to oral antibiotics. ${ }^{23,24}$ As treatment with antibiotic-corticosteroid eardrops is also clinically superior, less costly and avoids systemic adverse events, it should be first-line treatment in children with ATTO. ${ }^{7}$

\section{CTTO}

Idicula and colleagues investigated the microbial composition and the presence of biofilm fragments in 15 samples of children with persistent otorrhea and found that bacteria were present in 11 samples and 8 of these contained DNAB-II. ${ }^{25}$ This is relevant because previous in vitro and animal research has shown that targeting the DNAB-II protein of the biofilm could lead to a collapse of the 
biofilm structure and this could therefore be a new target for therapy of chronic or recurrent TTO. ${ }^{26,27}$

\section{Complications}

Systemic use of quinolones has been associated with detrimental effects on collagenous tissue.

Therefore, Alrwisan et al. assessed the incidence of persistent tympanic membrane perforations

(defined as perforations requiring tympanoplasty) after tympanostomy tube extrusion in a retrospective cohort of 96,595 children with tympanostomy tubes who had been treated with either neomycin/hydrocortisone, ofloxacin, ciprofloxacine/hydrocortisone or ciprofloxacine/dexamethasone eardrops. ${ }^{28}$ In children treated with quinolone eardrops the incidence was 17.3 per 10,000 person years as compared to 11.4 per 10,000 person years in those treated with neomycin/hydrocortisone eardrops. Despite being higher in the quinolone group, the incidence of a persistent perforation was very low which implies that clinicians need to balance this increased risk against the risk of sensorineural hearing loss caused by non-quinolone antibiotic eardrops or the ear infection itself.

\section{Conclusion}

Recent evidence shows that water precautions do not prevent children with tympanostomy tubes from developing TTO. It is important that clinicians take note of this evidence and advise parents and children accordingly.

The recommendation for eardrops as a first-line treatment in children presenting with ATTO is supported by recent evidence. Antibiotic-corticosteroid drops appear to be more effective than antibiotic-only drops. 
Quinolone eardrops are linked to a higher risk of persistent perforation of the eardrum after tympanostomy tube extrusion, though this risk needs to be balanced against potential ototoxicity of non-quinolone antibiotic eardrops and the ear infection itself. The clinical relevance of this finding however is up for debate.

\section{Key points}

- Water precautions do not protect children with tympanostomy tubes from developing TTO;

- Antibiotic-corticosteroid eardrop are most effective as the first line treatment of ATTOs.

\section{Acknowledgements}

None

\section{Financial support and sponsorship}

The authors did not receive funding for this work.

\section{Conflicts of Interest}

The UCL Ear Institute evidENT team is supported by various grants from the UK National Institute of Health Research. As director of the NIHR UCLH BRC Deafness and Hearing Problems Theme, Prof Anne GM Schilder advises on clinical trial design and delivery to a range of biotech companies. She is member of the Development Advisory Board of Novus Therapeutics. The remaining authors have no potential conflicts of interest to disclose.

\section{References}

1. Hall MJ, Schwartzman A, Zhang J, Liu X. Ambulatory surgery data from hospitals and ambulatory surgery centers: United States, 2010. National health statistics reports; no 102. Hyattsville, MD: National Center for Health Statistics. 2017. 
2. van Dongen TM, van der Heijden GJ, Freling HG, et al. Parent-reported otorrhea in children with tympanostomy tubes: incidence and predictors. PLoS One 2013;8:e69062.

3. Kay DJ, Nelson M, Rosenfeld RM. Meta-analysis of tympanostomy tube sequelae. Otolaryngol Head Neck Surg 2001;124:374-80.

4. Rosenfeld RM, Bhaya MH, Bower CM, et al. Impact of tympanostomy tubes on child quality of life. Arch Otolaryngol Head Neck Surg 2000;126:58592.

5. Moualed D, Masterson L, Kumar S, et al. Water precautions for prevention of infection in children with ventilation tubes (grommets). Cochrane Database of Systematic Reviews 2016, Issue 1. Art. No.: CD010375. DOI: 10.1002/14651858.CD010375.pub2.

6. van Dongen TM, van der Heijden GJ, Venekamp RP, et al. A trial of treatment for acute otorrhea in children with tympanostomy tubes. N Engl J Med 2014;370:723-33

7. van Dongen TM, Schilder AG, Venekamp RP, et al. Cost-effectiveness of treatment of acute otorrhea in children with tympanostomy tubes Pediatrics. 2015;135:e1182-9.

8. Rosenfeld RM, Schwartz SR, Pynnonen MA, et al. Clinical practice guideline: Tympanostomy tubes in children. Otolaryngol Head Neck Surg. 2013;149:S1-35

9. Damoiseaux RA, Venekamp RP, Eekhof JA, et al. NHG-Standaard Otitis media acuta bij kinderen [in Dutch]. Huisarts Wet 2014;57:648.

10. Poss JM, Boseley ME, Crawford JV. Pacific Northwest survey: posttympanostomy tube water precautions. Archives of Otolaryngology -- Head \& Neck Surgery 2008;134(2):133-5.

11. Ridgeon $\mathrm{E}$, Lawrence $\mathrm{R}$, Daniel M. Water precautions following ventilation tube insertion: what information are patients given? Our Experience. Clin Otolaryngol. 2016;41:412-6.

12. * Steele DW, Adam GP, Di M, et al. Prevention and Treatment of Tympanostomy Tube Otorrhea: A Meta-analysis. Pediatrics. 2017;139:e20170667. This is a comprehensive review including data on both prevention (including randomized as well as nonrandomized studies) as treatment (with a network meta-analysis).

13. Subtil J, Jardim A, Peralta Santos A, et al. Water protection after tympanostomy (Shepard) tubes does not decrease otorrhea incidence retrospective cohort study. Braz J Otorhinolaryngol. 2018;84:500-505.

14. Ricks RG, Easto R, Reddy VM. The water penetration of different ventilation tubes. Eur Arch Otorhinolaryngol. 2016;273:3131-4.

15. Ibrahim Y, Fram P, Hughes G, et al. Water penetration of grommets: an in vitro study. Eur Arch Otorhinolaryngol. 2017;274:3613-3617.

16. Tamir SO, Marom T, Zaks B, et al. Pseudomonal Biofilm Topographic Distribution on Tympanostomy Tubes: An In Vitro Model. Pediatr Infect Dis J. $2016 ; 35: 1357-1360$

17. Knutsson J, Priwin C, Hessén-Söderman AC, et al. A randomized study of four different types of tympanostomy ventilation tubes - Full-term followup. Int J Pediatr Otorhinolaryngol. 2018;107:140-144

18. Bizzell JG, Cox MD, Wang AR, et al. The impact of tobacco exposure on development of otorrhea after myringotomy tube placement. Int J Pediatr Otorhinolaryngol. 2017;92:67-69.

19. Park AH, White DR, Moss JR, et al. Phase 3 Trials of Thermosensitive Ciprofloxacin Gel for Middle Ear Effusion in Children with Tubes. Otolaryngol Head Neck Surg. 2016;155:324-31.

20. Dohar JE, Don D, Koempel J, et al. Safety and efficacy of intratympanic ciprofloxacin otic suspension post-tubes in a real-world pediatric population. Am J Otolaryngol. 2018;39:101-106

21. * Venekamp RP, Javed F, van Dongen TM, et al. Interventions for children with ear discharge occurring at least two weeks following grommet (ventilation tube) insertion. Cochrane Database Syst Rev. 2016;11:CD011684.

Comprehensive and thorough Cochrane review.

22. Spektor Z, Pumarola F, Ismail K, et al. Efficacy and Safety of Ciprofloxacin Plus Fluocinolone in Otitis Media With Tympanostomy Tubes in Pediatric Patients: A Randomized Clinical Trial. JAMA Otolaryngol Head Neck Surg. 2017;143:341-349. 
23. Ohyama M, Furuta S, Ueno K, et al. Ofloxacin otic solution in patients with otitis media: an analysis of drug concentrations. Arch Otolaryngol Head Neck Surg. 1999;125(3):337-340

24. Weber PC, Roland PS, Hannley M, et al. The development of antibiotic resistant organisms with the use of ototopical medications. Otolaryngol Head Neck Surg. 2004;130(3 Suppl):S89-94. doi:10.1016/j.otohns.2003.12.009.

25. Idicula WK, Jurcisek JA, Cass ND, et al. Identification of biofilms in post-tympanostomy tube otorrhea. Laryngoscope. 2016;126:1946-51.

26. Goodman SD, Obergfell KP, Jurcisek JA. Biofilms can be dispersed by focusing the immune system on a common family of bacterial nucleoidassociated proteins. Mucosal Immunology. 2011;4:625-637.

27. Brandstetter KA, Jurcisek JA, Goodman SD, et al. Antibodies directed against integration host factor mediate biofilm clearance from nasopore. Laryngoscope. 2013;123:2626-2632.

28. * Alrwisan A, Antonelli PJ, Winterstein AG. Quinolone Ear Drops After Tympanostomy Tubes and the Risk of Eardrum Perforation: A Retrospective Cohort Study. Clin Infect Dis. 2017;64:1052-1058.

Although the clinical relevance of the found association is unclear, this study using a large Medicaid cohort is exemplary for future studies on rare complications of medication. 